« Clinique »

\title{
SUICIDE ET EVALUATION. REVUE DES OUTILS DISPONIBLES EN FRANÇAIS : APPROCHE NON DIMENSIONNELLE ET AUTO-QUESTIONNAIRES.
}

\section{J.L. Ducher (a), Ingrid de Chazeron (b), P.M. Llorca (c)}

(a) Clinique de l'Auzon - 63670 La Roche-Blanche

(b) Clermont Université, Université d'Auvergne, EA 4681, PEPRADE ; CHU ClermontFerrand, Service de Psychiatrie B, F-63003 Clermont-Ferrand.

(c) Clermont Université, Université d'Auvergne, EA 7280, CHU Clermont-Ferrand, Service de Psychiatrie B, F-63003 Clermont-Ferrand.

Auteur correspondant :

J.L. Ducher

Clinique de l'Auzon - 63670 La Roche-Blanche

04.73.35.50.18 - 04.73.79.84.46

Jean-luc.ducher@hotmail.fr

Reçu le 9 Octobre 2013

Accepté le 27 novembre 2014 
Titre : SUICIDE ET EVALUATION. REVUE DES OUTILS DISPONIBLES EN FRANÇAIS: APPROCHE NON DIMENSIONNELLE ET AUTO-QUESTIONNAIRES.

Mots-clés : Suicide ; tentative de suicide ; risque suicidaire ; évaluation ; échelles.

Résumé : La prévention du suicide représente un enjeu majeur de santé publique, le risque suicidaire un souci permanent en psychiatrie. La difficulté principale en est son diagnostic. Quels sont les moyens disponibles en français qui semblent pouvoir aider les thérapeutes dans cette démarche ? Nous pouvons distinguer l'approche dimensionnelle, que ce soit à travers l'utilisation d'auto-questionnaires ou d'hétéro-questionnaires, et l'approche non dimensionnelle. Dans cet article, pour des raisons de contraintes éditoriales, nous nous sommes intéressés uniquement à l'approche non dimensionnelle et aux mesures par autoévaluation, en analysant les atouts et les limites des unes et des autres ainsi qu'en tenant compte des études scientifiques qui leur ont été consacrées et de leur intérêt clinique.

Nous avons d'abord considéré différents aspects de l'approche non dimensionnelle à travers l'intérêt de la recherche des facteurs de risque, les concepts d'urgence et de potentiel suicidaires, la démarche de Shea, le modèle de Mann et certaines recommandations d'évaluation proposées. Cette démarche possède un certain nombre d'avantages, mais aussi de limites.

L'approche dimensionnelle permet d'aller plus loin. Dans cet article, nous abordons aussi les outils d'auto-évaluation existant en français qu'ils soient sous la forme d'item dédié comme dans l'Inventaire de Dépression de Beck (BDI) ou d'échelles spécifiques comme l'Inventaire des Raisons de Vivre (IRV), l'Echelle de Probabilité Suicidaire (SPS), l'échelle de Désespoir de Beck (BHS) ou l'échelle d'auto-évaluation du Risque Suicidaire de Ducher (aRSD). Ces deux dernières semblent devoir être utilisées en priorité en raison des études de validation dont elles ont fait l'objet. La forte corrélation existant entre l'auto-questionnaire aRSD et l'hétéro-questionnaire d'évaluation du Risque Suicidaire de Ducher RSD ( $\left.\mathrm{r}=0.92 ; \mathrm{p}<10^{-7}\right)$ montre bien la capacité des patients à exprimer leurs idéations suicidaires pour peu qu'on les invite à le faire. 
Title: SUICIDE AND EVALUATION. REVIEW OF FRENCH TOOLS: NONDIMENSIONAL APPROACH AND SELF-ASSESSMENT.

Keywords: Suicide; suicide attempt; suicide risk; assessment; rating scales.

Summary: Suicide prevention represents a major challenge to public health and the suicide risk is a permanent concern in psychiatry. But the main difficulty is its diagnosis. What resources are available in French, which seem to help therapists in this process? We can distinguish the non-dimensional approach, the use of self-administered questionnaires or interviewer-administrated questionnaires. In this paper, for reasons of editing constraints, we are interested only in non-dimensional approach and direct assessment measures by selfassessment, analyzing the strengths and limitations of each and taking into account scientific studies that have been devoted to them and their clinical relevance.

We first considered various aspects of non-dimensional approach through suicidal risk factors research, suicidal emergency and suicidal potential concepts, Shea approach, the model of Mann and some recommended evaluations. This type of approach has a number of advantages, but also limitations.

Par exemple, de nombreux facteurs de risque ont été rapportés tant en ce qui concerne le suicide que les tentatives de suicide. Le problème est qu'en pratique, ils permettent plus de déterminer si un patient appartient à une population à risque que de savoir si celui-ci présente un risque de passage à l'acte à un moment précis. Mann met en avant plusieurs facteurs de risque indépendants des troubles psycho-pathologiques sous-jacents, en particulier la propension à l'impulsivité ou à l'agressivité.

Le concept de potentiel suicidaire développé au Canada dans les années 1970-80, alliant la mesure du risque suicidaire et de l'urgence suicidaire n'a jamais été validé et est de ce fait de plus en plus abandonné.

La Conférence de Consensus sur le suicide ainsi que le référentiel de I'HAS sur la Prise en charge d'un épisode dépressif proposent des évaluations basées sur de nombreux éléments tout à fait intéressants 


\section{d'un point de vue clinique, mais dont le nombre rend la lecture globale difficile.}

Dimensional approach allows going further. In this article, we also discuss the existing selfassessment tools in French as for example dedicated item for Beck Depression Inventory (BDI) or specific scales such as Reasons for Living Inventory (RFL), Suicidal Probability Scale (SPS), Beck Hopelessness Scale (BHS) and self-administered Suicide Risk assessment scale of Ducher (aRSD). These last two seem to be used as a priority regarding result of their validation studies. The strong correlation between the self-administered questionnaire aRSD and the interviewer-administered Suicide Risk assessment scale of Ducher RSD ( $\mathrm{r}=0.92 ; \mathrm{p}<$ $10^{-7}$ ) shows the ability of patients to express their suicidal ideation, if we want to invite them to do so. 


\section{SUICIDE ET EVALUATION. REVUE DES OUTILS DISPONIBLES EN FRANÇAIS : APPROCHE NON DIMENSIONNELLE ET AUTO-QUESTIONNAIRES.}

Comment comprendre l'absence de diminution du nombre de suicides et l'augmentation considérable de celui des tentatives de suicide en France ces dernières années, malgré la mise en place de nombreuses actions de prévention [1] ? Une des explications à envisager est le non-respect d'une règle simple : face à un patient dépressif, l'évaluation du risque suicidaire doit être systématique, de même que face à toute personne susceptible de présenter un tel risque, quel que soit la pathologie dont elle souffre. Encore faut-il que le soignant soit formé pour cela et capable d'utiliser des outils efficaces. La simple impression clinique ne semble pas suffisante [2]. L'étude de Appleby et coll. portant sur 2170 patients psychiatriques décédés par suicide, montre que $20 \%$ de ces personnes avaient été en contact avec les services de santé mentale 24 heures avant leur décès et $50 \%$ dans les 8 jours précédant. Leur risque suicidaire avait été évalué comme absent ou faible dans $84 \%$ et élevé dans seulement $2 \%$ des cas [3]. Le problème ne concerne pas seulement le cadre des consultations ou des services d'urgence. L'incidence du suicide est de 250 pour 100000 admissions en milieu psychiatrique et de 1,8 pour 100000 admissions dans les hôpitaux généraux (quatre à cinq fois plus qu'en population générale). Trois à 5,5\% des suicides sont ainsi commis en hôpital psychiatrique et environ $2 \%$ en hôpital général [4].

Contrairement à l'inquiétude exprimée par certains professionnels de santé, la conférence de consensus sur la crise suicidaire déclare qu'il ne faut pas « hésiter à questionner les patients sur leurs idées de suicide, cette attitude loin de renforcer le risque suicidaire ne peut que favoriser l'expression des troubles...» [5].

De plus, le référentiel d'auto-évaluation de prise en charge d'un état dépressif isolé (Critère 3) fait obligation de mentionner dans le dossier médical cette évaluation du risque suicidaire à chaque consultation, aussi longtemps que l'état du patient le justifie [6]. En milieu hospitalier, l'inscription de cette évaluation dans le dossier du patient plusieurs fois par semaine semble donc justifiée.

Reste à savoir comment la réaliser ? De nombreux moyens plus ou moins efficaces ont été décrits. Nous pouvons les séparer en trois groupes: l'approche non dimensionnelle, l'évaluation dimensionnelle par auto-questionnaires et celle par hétéro-questionnaires. Dans le cadre cet article, en raison des contraintes d'édition, nous ne pourrons nous intéresser qu'aux deux premiers. 


\section{Méthodologie}

A cette fin, nous avons effectué une revue de la littérature par l'interrogation exhaustive des bases de données PubMed®, Medline ${ }^{\circledR}$ et Google ${ }^{\circledR}$ en utilisant les termes « suicide scale », « suicide questionnaire », « suicide evaluation », « suicide risk ». Plusieurs itérations ont été faites, une première sur des mots-clés et leur traduction française, une deuxième sur la lecture des résumés d'articles. Une étape supplémentaire a consisté à résumer et à coder les références afin de les regrouper. Un critère de sélection majeur a été l'existence de version française validé. Un tableau de références a ainsi été établi selon la méthode de Hart [7].

\section{LES APPROCHES NON DIMENSIONNELLES}

Si cette démarche n'autorise que rarement une appréciation très précise du risque de passage à l'acte pour en permettre un suivi régulier, elle permet souvent de distinguer des populations à risque pour lesquelles une attention particulière doit être apportée.

\section{$\underline{\text { A/ Les facteurs de risque }}$}

Même si de nombreux patients expriment clairement leur intention avant de passer à l'acte, certains auteurs estiment qu'il n'est pas possible de prédire un tel comportement et qu'on ne peut que repérer des facteurs de risque. De nombreuses études identifient des facteurs associés à un plus grand risque soit de décès par suicide, soit de tentatives de suicide. Mais cette approche reste limitée, en particulier en raison du nombre de facteurs décrits (plus de 100), ce qui en complexifie l'analyse. De nombreuses études, incluant souvent plusieurs milliers de patients avec un suivi sur de nombreuses années, n’ont pu retrouver de spécificité très fiable pour pratiquement aucun d'entre eux. Dans leur étude prospective sur 8 ans de 200 patients hospitalisés en psychiatrie, Bioulac, M Bourgeois et coll. n'ont retrouvé de façon significative dans le groupe des suicidés ( $5 \%$ de décès par suicide), parmi les différents facteurs réputés prédictifs, que les antécédents familiaux (1er degré) de suicide et d'hospitalisation psychiatrique, et l'impulsivité (peut-être liée à l'âge) [8].

Mais Rihmer [9] rappelle que plus des deux tiers de victimes de suicide meurent dès leur première tentative et insiste donc sur la nécessité de détecter des facteurs de risque de suicide, particulièrement chez les patients présentant des troubles de l'humeur, afin d'intervenir avant que la personne ne passe à l'acte. 
D’une manière générale, on distingue [5] :

- Les facteurs primaires : troubles psychiatriques, antécédents personnels et familiaux de suicide, communication d'une intention suicidaire ou tendance à l'impulsivité... Ils représentent une valeur d'alerte importante au niveau individuel et peuvent en général être influencés par le traitement.

- Les facteurs secondaires : pertes parentales précoces, isolement social, chômage, difficultés financières et professionnelles, événements de vie négatifs... Leur valeur prédictive s'avère faible en l'absence de facteurs primaires et ils sont peu modifiables par le traitement.

- Les facteurs tertiaires : appartenance au sexe masculin, au grand ou au jeune âge, à une période de vulnérabilité (phase prémenstruelle)... Ils n'ont de valeur prédictive qu'en présence de facteurs primaires et secondaires et restent non modifiables par le traitement.

Certains facteurs de risque semblent prendre une importance prépondérante en fonction des circonstances. Par exemple, au cours d'une première semaine d'hospitalisation en psychiatrie, on va retrouver les antécédents suicidaires personnels et familiaux, les tentatives de suicide (TS) peu avant l'admission, l'existence d'un trouble schizophrénique ou de l'humeur, une comorbidité alcoolique, une hospitalisation sans consentement, le fait de vivre seul, de s'être absenté du service sans permission. Au décours de l'hospitalisation, ce sera davantage les antécédents suicidaires personnels, les idées suicidaires ou de TS peu avant l'admission, une tentative de suicide durant l'hospitalisation, l'existence de difficultés relationnelles, d'un stress professionnel, une perte d'emploi, le fait de vivre seul, une décision de sortie de l'hôpital non planifiée et l'absence de contact avec des soignants après la sortie. En hôpital général, on cite le plus souvent la chronicité et la sévérité de la maladie somatique, la personnalité du patient et l'existence d'une comorbidité psychiatrique [4].

\section{B/ Le modèle explicatif de Mann}

Mann et coll. [10] se sont aussi intéressés à la recherche de facteurs de risque prédictifs du suicide, mais leur démarche est partie d'une hypothèse théorique sur la psychopathologie du suicide particulière en suggérant une prédisposition spécifique aux conduites suicidaires, indépendante des troubles psychiatriques éventuellement associés. Ils ont séparé des sujets présentant différents troubles psychiatriques en deux groupes en fonction de l'existence d'antécédents suicidaires ou non. Ils constatent que ceux qui en présentent, semblent éprouver plus de désespoir et d'idéations suicidaires, perçoivent moins de raisons de vivre, malgré une gravité objective du trouble psychiatrique et un nombre d'événements de vie négatifs 
similaires à l'autre groupe. Ils présentent également une propension à l'impulsivité et à l'agressivité plus marquée.

Cette démarche est d'autant plus intéressante que ces facteurs peuvent également faire l'objet d'une évaluation dimensionnelle indépendante grâce à des échelles spécifiques. Par exemple, bien qu'il s'agisse d'un concept controversé, l'impulsivité peut être appréciée par l'échelle d'impulsivité de Barratt [11] qui a été le premier à en proposer une ou par celle d'impulsivité non-conformité de Chapman [12].

Cependant, même si un patient présente des facteurs de risque, rien ne permet d'imaginer l'imminence éventuelle de son passage à l'acte. De plus, nombre de personnes ayant plusieurs facteurs de risque ne passent pas à l'acte et à l'inverse, certaines qui en sont indemnes, le font.

\section{$\underline{\text { C/ L'urgence suicidaire }}$}

Pour compenser ce manque d'informations sur la proximité d'un éventuel passage à l'acte, la Conférence de consensus citée plus haut énonce trois niveaux d'urgence : faible, moyen ou élevé, en fonction du comportement, de l'état émotionnel, de celui de souffrance, voire de désarroi, des projets de vie, de la capacité conservée ou perdue à envisager des solutions à ses problèmes, des idéations ou du scénario suicidaires, de l'accès au moyen projeté...

Malgré son intérêt clinique évident, cette démarche possède une forte complexité en raison du nombre important d'éléments à prendre en considération et aussi des réponses parfois contradictoires qui peuvent leur être associées. Ceci rend l'appréciation de l'urgence suicidaire et la répétition de son évaluation pas toujours aussi simples qu'il serait souhaitable pour garantir une bonne fiabilité. Une formation et une bonne pratique clinique sont nécessaires.

\section{$\underline{\text { D/ Le potentiel suicidaire }}$}

Dans les années 1970-80, un certain nombre d'auteurs canadiens ont élaboré le concept de « potentiel suicidaire » qui comprend à la fois la mesure du risque suicidaire et de l'urgence suicidaire. Parmi eux, Pierre Morissette définit le risque suicidaire comme «la probabilité ou la possibilité que la personne qui signale son intention, passe à l'acte de façon mortelle, dans les deux prochaines années ». L'urgence suicidaire évalue cette même probabilité pour les prochaines 48 heures [13].

Le risque suicidaire dépend de facteurs sociaux, environnementaux, liés à l'individu ou aux circonstances. Il ne change pas à court, ni moyen terme, alors que l'urgence suicidaire évolue constamment. Son appréciation nécessite l'évaluation des idéations, des intentions suicidaires, 
d'une planification éventuelle... «En fait, à cause de l'aspect temporel lié à ces mesures, le risque et l'urgence sont très différents et n'ont qu'un rapport lointain entre eux». Cette démarche s'oppose à l'approche américaine qui parle de «suicide risk» sans faire cette distinction.

Morissette lui-même, écrit que l'urgence suicidaire «correspond à des critères d'évaluation moins précis que le risque suicidaire. L'évaluation de l'urgence est plus subjective ; elle fait appel au jugement, à l'attention et à l'expérience de l'intervenant ». Cette approche ne permet donc que difficilement de suivre l'évolution de la crise suicidaire et peut donner un faux sentiment de sécurité.

Le problème majeur de ce concept se retrouve dans le fait qu'aucune étude n'en a jamais démontré la validité. La clinique montre bien que la prise de décision et le passage à l'acte peuvent parfois se faire très rapidement chez un patient dépressif, même non impulsif et sans facteurs de risque.

\section{E/ Évaluation chronologique des évènements suicidaire (Méthode ECES)}

Dans leur livre [14], Shea et all. critiquent fortement la démarche qui consiste à rechercher des facteurs de risque en remarquant qu'aucun facteur de risque statistique ne pourra indiquer si tel ou tel patient a l'intention de mourir, certains pouvant par ailleurs se tuer accidentellement. Ils préfèrent insister sur la démarche d'interrogation du patient et se concentrer sur la recherche d'évènements suicidaires éventuels (idées ou conduites) au cours des différents temps qui ont précédé la consultation (méthode ECES) : événements actuels, récents (<2 mois), passés, immédiats (intention suicidaire). Si cette démarche n'est pas validée, elle ne manque pas d'intérêt clinique: «M. Jones, aidez-moi à comprendre exactement ce qui s'est passé lors de votre intoxication médicamenteuse. Essayer de me décrire étape par étape la nuit dernière. Commençons par le moment où vos idées de suicides sont devenues plus fortes. »

\section{$\underline{\text { F/ Les critères du référentiel de la prise en charge d'un épisode dépressif isolé (HAS) }}$}

Entre une démarche essentiellement clinique et une recherche exclusive des facteurs de risque, quelle attitude adopter ? Le référentiel sur la prise en charge d'un épisode dépressif isolé [6] recommande pour reconnaitre et évaluer le risque suicidaire d'explorer 6 éléments : le niveau de souffrance, le degré d'intentionnalité, les éléments d'impulsivité, un éventuel élément précipitant, la présence de moyens létaux à disposition, la qualité du soutien de l'entourage proche. Chacun de ces différents éléments s'intéresse lui-même à plusieurs 
aspects. Par exemple, le niveau de souffrance s'apprécie à travers le désarroi ou le désespoir, le repli sur soi, les sentiments de dévalorisation, d'impuissance, de culpabilité...

La tendance à augmenter le nombre de critères explorés peut donner un sentiment plus grand de sécurité et d'efficacité, mais malheureusement, elle complique souvent l'interprétation des données et peut en fausser l'analyse. Et que faire lorsque les réponses à plusieurs critères pertinents s'opposent?

C'est pour cela qu'il peut être utile de se tourner vers l'approche dimensionnelle qui explore de manière plus précise une dimension spécifique du problème. Celle-ci peut utiliser des autoou des hétéro-questionnaires. Pour des raisons d'édition, nous nous intéresserons dans cet article qu'aux échelles d'auto-évaluation.

\section{LES ECHELLES D’AUTO-EVALUATION}

L'utilisation d'échelles permet de structurer l'évaluation du risque suicidaire et d'instaurer une intervention souvent plus appropriée. Il existe une grande variabilité des instruments de mesure. Les auto-questionnaires peuvent se présenter sous la forme :

- soit de sous-échelle ou d'item dédié d'une échelle plus globale, généralement de dépression,

- $\quad$ soit d'échelles spécifiques.

\section{A / Items dédiés ou Sous-échelles}

Assez logiquement, les échelles d'auto ou d'hétéro-évaluation de la dépression possède un «item suicide ». Il est parfois utilisé de manière indépendante pour évaluer le risque suicidaire.

\section{$\underline{\text { L'inventaire de dépression de Beck: item suicide }}$}

La Beck Depression Inventory (BDI) [15] est une des échelles d'auto-évaluation de la dépression les plus répandues. Elle comprend 21 items qui peuvent se regrouper en deux souséchelles: une portant sur le plan cognitivo-affectif (items 1 à 13), l'autre sur celui du ressenti somatique (items 14 à 21) [16]. Une version française existe [17], ainsi qu'une forme abrégée correspondant à la sous-échelle cognitivo-affective (BDI-SF) et une version plus récente, la BDI-II [18], modifiée afin de tenir compte des critères du DSM-IV. Comme la BDI, la BDI-II comporte 21 items, mais les items qui traitent de perte de poids, des changements de l'image 
corporelle, de la préoccupation somatique, de la difficulté du travail, du sommeil et de l'appétit ont été modifiés.

La BDI et ses dérivés n'ont pas été spécifiquement conçus pour mesurer le risque suicidaire, mais sont largement utilisés comme échelles d'auto-évaluation pour apprécier la sévérité d'un état dépressif. Le score total à la BDI ne possède qu'une faible relation avec le risque de passage à l'acte suicidaire $[10,19]$. La BDI possède un item suicide, coté de 0 à 3 , mais il varie dans sa formulation selon les différentes versions existant (Je ne pense pas à me faire du mal / je pense que la mort me libérerait / J'ai des plans précis pour me suicider / Si je le pouvais, je me tuerais : pour la BDI version abrégée 13 items). Dans la version longue à 21 items, les degrés 1 et 2 de la version courte sont cotés de manière identique à 2 . Reste donc à savoir quelle version utiliser de manière préférentielle. L'étude de Desseilles et coll. [20] s'est intéressée aux corrélations existant entre l'item «suicide» de la BDI version abrégée, l'échelle Hamilton Dépression et l'échelle d'Idéation Suicidaires de Beck.

D'un point de vue clinique, on peut cependant regretter le faible éventail d'évaluation de cet item, avec en particulier l'absence de possibilité de quantification des idéations suicidaires, ce qui ne pose pas de réelles difficultés lorsqu'on désire évaluer l'intensité d'un état dépressif, mais reste beaucoup plus problématique lorsqu'on veut réaliser le suivi précis du risque suicidaire d'un patient.

\section{$\underline{B / \text { Echelles spécifiques }}$}

Des échelles spécifiques existent, mais abordent le plus souvent l'évaluation du risque suicidaire de manière indirecte, à travers un aspect particulier de la dimension suicidaire, tel la recherche des raisons de vivre du patient, son niveau de désespoir, son évaluation négative de lui-même, son degré d'hostilité. Seule l'auto-questionnaire aRSD interroge le patient uniquement et directement sur son risque suicidaire.

\section{1/ L'Inventaire des Raisons de Vivre (IRV)}

L'auto-questionnaire Reasons for Living Inventory (RFL) de Linehan et coll. [21, 22] a été traduit en langue française par Labelle et coll. en1996. Il comprend 48 questions cotées selon une échelle de Likert à 6 points, allant de (1) «très peu d'importance » à (6) « extrêmement importante », qui évaluent les facteurs de protection possibles chez des personnes disant avoir des idées suicidaires. L'analyse factorielle a donné au moins 6 sous-échelles distinctes [22, 23]: 'Survie et croyances d'adaptation', ' Responsabilité familiale', 'Préoccupations des 
enfants', 'Peur du suicide', 'Peur de la désapprobation sociale' et 'Objections morales'. Les six sous-échelles se fondent sur quatre analyses factorielles menées sur deux échantillons d'adultes normaux volontaires [22]. Une analyse factorielle confirmatoire subséquente n'a constaté qu'un appui modéré pour le modèle à six facteurs chez les patients psychiatriques [24].

L'IRV est fondée sur une vision comportementale et cognitive du comportement suicidaire qui postule que les schémas cognitifs, les croyances, les attentes ou les cognitions, peuvent être des médiateurs importants des comportements suicidaires. Un aspect très spécifique de l'IRV est sa formulation positive. Selon Range et Knott [25], son remplissage pourrait avoir un impact préventif dans le suicide. Sa passation prend environ une dizaine de minutes et même si une corrélation négative a été retrouvée avec l'échelle d'idéations suicidaires de Beck (SSI), aucune donnée probante ne permet de confirmer sa valeur prédictive.

\section{$\underline{\text { 2/ Suicide Probability Scale (SPS) }}$}

L'échelle de probabilité du suicide [26], utilisable chez les adultes et les adolescents, a été traduite en langue française par Labelle et coll. en 1998 [27]. Elle se compose de 36 items (ex : "Je me sens isolé par rapport aux autres") parmi 200 considérés initialement, selon les auteurs, comme appartenant aux quatre plus importants indicateurs de suicide, à savoir : l'anomie (Durkheim), la colère introspective (Freud), la létalité - la perturbation et le comportement inamical (Shneidman) et les observations cliniques générales de l'impulsivité (Golding). Les items sont cotés en fonction de la fréquence durant laquelle la personne se sent concernée (de 'jamais ou quelques fois' à 'la plupart du temps ou toujours'). L'échelle se décompose en 4 sous-échelles : 'Désespoir' (12 items), 'Idéations suicidaires' actuelles (6 items), 'Auto-évaluation négative' (9 items), 'Hostilité' incluant l'impulsivité et la colère (9 items). Le questionnaire SPS demande entre 10 et 20 minutes pour le remplir. Même si plusieurs études ont analysé les qualités psychométriques de cette échelle, des investigations supplémentaires semblent souhaitables et certains recommandent son utilisation avec précaution [28]. Il semble en particulier difficile de définir un score seuil de probabilité de suicide et la poursuite des investigations parait justifiée avant de pouvoir en recommander un [29]. 
De nombreuses études ont montré les relations existant entre le désespoir et le risque suicidaire. On peut citer en particulier l'étude de Chabrol et coll. [30] qui présente l'intérêt de s'adresser à des lycéens. Le désespoir y apparait comme un prédicteur significatif indépendant des idées suicidaires, jouant un rôle de médiateur entre les symptômes dépressifs et le désir de suicide.

L'Echelle de désespoir de Beck est un auto-questionnaire qui évalue le niveau d'espoir ou plutôt de désespoir à propos de l'avenir, la perte de motivation et les attentes futures. Il a été validé à l'origine auprès de 294 patients qui avaient tenté de se suicider [31, 32]. Sa cotation va de 0 à 20 . Il comporte 20 items avec neuf qui reflètent le pessimisme s'ils sont cotés « faux » et 11 , s'ils sont cotés « vrai ».

Une étude [33] sur 207 patients hospitalisés pour idéations suicidaires, sans TS récentes, suivis pendant 5 à 10 ans, a retrouvé que l'échelle BHS avait une «bonne corrélation » avec le suicide. S'il s'agit bien d'une étude prospective, la validation du score seuil n'a été faite que de manière rétrospective. Un score $\geq 10$ a ainsi identifié correctement $91 \%$ des 14 patients décédés dans l'étude. Mais les auteurs parlent de «the eventual suicides » car l'acte suicidaire n'a pas pu être certifié dans la totalité de ces décès.

Une validation en français a été réalisée par Bouvard et coll. [34]. Elle a montré une bonne corrélation avec le questionnaire des pensées automatiques ( $\mathrm{r}=0.78, \mathrm{p}<0.001)$, l'échelle d'attitudes dysfonctionnelles $(r=0.66, p<0.001)$, l'échelle d'évaluation du Risque Suicidaire de Ducher $(r=0.64, \mathrm{p}<0.001)$ et une plus faible avec l'échelle de dépression de Hamilton 17 $(\mathrm{r}=0.28, \mathrm{p}<0.005)$.

Le désespoir mesuré semble être un marqueur prédictif du comportement suicidaire pour les patients atteints de divers troubles psychiatriques $[35,36]$. Il a été montré qu'il possède une meilleure prédictivité du risque suicidaire que la mesure de la dépression [37, 38]. Cet outil a été utilisé dans des populations psychiatriques, mais aussi en population générale [39-41]. McMillan et coll. [42] estiment que la BHS pourrait être un bon outil d'évaluation initiale afin d'identifier les personnes qui ont besoin d'une évaluation clinique du risque suicidaire plus complète. Cependant Cochrane-Brink et coll. [43] ne conseillent pas son utilisation pour identifier les personnes à très haut risque d'automutilation non suicidaire répétée, ni pour mener une évaluation aux urgences. 
L'auto-questionnaire d'évaluation du Risque Suicidaire aRSD est basé sur le même principe que l'hétéro-questionnaire RSD [44,45].

Il possède également 1 seul item avec 11 modalités hiérarchisées. Il s'agit d'une échelle qui évalue entre 0 et 10 le niveau décisionnel dans lequel un patient est face à un éventuel désir de se donner la mort. Entre 1 et 5, le patient pense à la mort, au suicide, mais n'a pas envie de mourir. Entre 6 et 10, non seulement il a des idées de mort ou de suicide, mais en plus, il souhaite mourir, d'abord de manière passive (degré 6), puis retenue (degré 7), enfin décidée (degré 8), programmée (degré 9) ou actée (degré 10).

Une étude sur 100 patients des deux sexes, présentant un épisode dépressif majeur, hospitalisés ou non, a démontré une corrélation très significative entre l'auto-questionnaire aRSD et l'hétéro-questionnaire RSD $\left(\mathrm{r}=0.92 ; \mathrm{p}<10^{-7}\right)$. En analyse appariée, aucune différence ne sépare les deux questionnaires $(\mathrm{p}=0.87)$. De manière assez remarquable, ceci montre que ces deux échelles, auto-administrées ou cotées par un évaluateur externe, mesurent pratiquement la même chose [46].

\section{Conclusion}

Les patients suicidaires parlent souvent de leurs idées, voire de leur intention, à leur entourage ou consultent avant de passer à l'acte. Il faut donc savoir être attentif à ces différents comportements, en particulier face à des patients dépressifs chez qui l'évaluation du risque suicidaire doit être répétée systématiquement et inscrite dans le dossier médical. Reste à savoir comment apprécier ce risque.

L'approche non dimensionnelle avec en particulier la recherche de facteurs de risque permet d'identifier des populations précises qui peuvent bénéficier d'un suivi plus attentif, mais pas de savoir le risque immédiat éventuel d'un patient. La multiplication des facteurs de risque, dans un but recherché d'une plus grande efficacité, complexifie souvent cette appréciation. Cependant, il faut savoir tenir compte des critères définis par Mann et coll. (impulsivité, agressivité en autre) qui semblent être des facteurs indépendants des troubles psychiatriques sous-jacents.

L'approche dimensionnelle semble souvent plus pertinente. Mais la validation d'une échelle concernant le suicide reste toujours difficile en raison du biais que représente la priorité donnée à la protection de la personne évaluée. Peu d'échelles ont donc fait l'objet de telles études. Celles-ci doivent être utilisées de manière préférentielle. On peut citer comme auto- 
questionnaires entrant dans ce cadre l'échelle de Désespoir de Beck et l'échelle d'autoévaluation du Risque Suicidaire de Ducher.

Dans cet article, pour des raisons de contraintes éditoriales, nous nous sommes seulement intéressés dans le cadre de l'approche dimensionnelle aux instruments d'auto-évaluation, mais l'emploi d'échelles d'hétéro-évaluation se révèle souvent d'un grand intérêt. Là encore, la priorité doit être donnée aux instruments ayant fait l'objet d'étude de validation. Plusieurs peuvent être cités : l'Échelle d'idéations suicidaires de Beck (SSI), l'échelle d'évaluation du Risque Suicidaire de Ducher (RSD), l'échelle de l'équipe de Kelly Posner et John Mann (CSSRS : Columbia-Suicide Severity Rating Scale).

\section{Conflit d'intérêt : aucun.}

\section{REFERENCES}

[1] Ducher JL, Llorca PM. [No! The number of suicides in France is not decreasing]. Encéphale. $2012 ; 38(5): 371-2$.

[2] Ducher JL, Dalery J. [Correlations between Beck's suicidal ideation scale, suicidal risk assessment scale RSD and Hamilton's depression rating scale]. Encéphale 2008 ;34(2):132-8.

[3] Appleby L, Shaw J, Amos T, et al. Suicide within 12 months of contact with mental health services: national clinical survey. BMJ. 1999 ;318(7193):1235-9.

[4] Martelli C, Awad H, Hardy P. [In-patients suicide: epidemiology and prevention]. Encéphale $2010 ; 36$ Suppl 2:D83-91.

[5] Fédération française de psychiatrie. [La crise suicidaire : reconnaître et prendre en charge]. John Libbey Eurotext ed ; 2001.

[6] Haute Autorité de Santé, Service évaluation des pratiques. Référentiel d'auto-évaluation ds pratiques en psychiatrie 2005. Available from: http://www.hassante.fr/portail/upload/docs/application/pdf/2010-08/epp cro generique ortho rap 201008-17 15-06-11 148.pdf.

[7] Hart C. Doing a literature review : Releasing the social science research imagination. LA/London: SAGE Publications Ltd; 2009.

[8] Bioulac S, Bourgeois M, Ekouevi DK, et al. [Predictive factors of suicide? an 8-year-long prospective longitudinal study of 200 psychiatric inpatients]. Encéphale. 2000 ;26(1):1-7.

[9] Rihmer Z. Suicide risk in mood disorders. Curr Opin Psychiatry 2007 ;20(1):17-22.

[10] Mann JJ, Waternaux C, Haas GL, et al. Toward a clinical model of suicidal behavior in psychiatric patients. Am J Psychiatry $1999 ; 156(2): 181-9$.

[11] Bayle FJ, Bourdel MC, Caci $\mathrm{H}$, et al. [Factor analysis of french translation of the Barratt impulsivity scale (BIS-10)]. Can J Psychiatry $2000 ; 45(2): 156-65$.

[12] Foullu S, Danet F, Dumas $P$, et al. [Validation of the French translation of the impulsive nonconformity scale]. Encéphale $2008 ; 34(6): 563-9$.

[13] Morissette P. [Le Suicide: démystification, intervention, prévention : mythes, tabous et réalités]. Québec: Centre de Prevention du Suicide; 1984.

[14] Shea S-C, Terra J-L, Séguin M, et al. [Evaluation du potentiel suicidaire : Comment intervenir pour prévenir]. Paris : Elsevier; 2008.

[15] Beck AT, Ward CH, Mendelson $\mathrm{M}$, et al. An inventory for measuring depression. Arch Gen Psychiatry $1961 ; 4: 561-71$.

[16] Beck AT, Steer RA. Beck Depression Inventory Manual. San Antonio, TX : Psychological Corporation ; 1993.

[17] Delay J, Pichot $P$, Lempériere $T$, et al. [The nosology of depressive states. Relation between etiology and semiology. 2. Results of Beck's questionnaire]. Encéphale. 1963 ;52:497-504. 
[18] Beck AT, Steer RA, Ball R, et al. Comparison of Beck Depression Inventories -IA and -II in psychiatric outpatients. J Pers Assess. 1996 ; 67(3):588-97.

[19] Lester D, Beck AT. Suicidal wishes and depression in suicidal ideators: a comparison with attempted suicides. J Clin Psychol. 1977 ;33(1):92-4.

[20] Desseilles M, Perroud N, Guillaume $S$, et al. Is it valid to measure suicidal ideation by depression rating scales? J Affect Disord. 2012 ;136(3):398-404.

[21] Labelle R, Lachance L, Morval M. French-Canadian version of the Reasons for Living Inventory. Science et Comportement 1996;24(3):237-48.

[22] Linehan MM, Goodstein JL, Nielsen SL, et al. Reasons for staying alive when you are thinking of killing yourself: the reasons for living inventory. J Consult Clin Psychol. 1983 ;51(2):276-86.

[23] Osman A, Gifford J, Jones T, et al. Psychometric evaluation of the Reasons for Living Inventory. Psychological Assessment 1993;5:154-58.

[24] Osman A, Downs WR, Kopper BA, et al. The Reasons for Living Inventory for Adolescents (RFL-A): development and psychometric properties. J Clin Psychol. 1998 ;54(8):1063-78.

[25] Range LM, Knott EC. Twenty suicide assessment instruments: evaluation and recommendations. Death Stud. 1997 ;21(1):25-58.

[26] Cull JG, Gill WS, editors. Suicide Probability Scale Manual. Los Angeles ; 1990.

[27] Labelle R, Daigle MS, Pronovost J, et al. [Étude psychométrique d'une version française du " Suicide Probability Scale " auprès de trois populations distinctes]. Psychologie et psychométrie 1998;19(1):4-27.

[28] CRISE (2008). Application des connaissances scientifiques en prévention du suicide. Available from: http://www.criseapplication.uqam.ca.

[29] Ontario Hospital Association. Guide d'évaluation du risque de suicide. Une ressource pour les organismes de santé ; 2012.

[30] Chabrol H, Choquet M. [Relationship between depressive symptoms, hopelessness and suicidal ideation among 1547 high school students]. Encéphale 2009 ;35(5):443-7.

[31] Beck AT, Weissman A, Lester D, et al. The measurement of pessimism: the hopelessness scale. J Consult Clin Psychol. $1974 ; 42(6): 861-5$.

[32] Hill RD, Gallagher D, Thompson LW, et al. Hopelessness as a measure of suicidal intent in the depressed elderly. Psychol Aging $1988 ; 3(3): 230-2$.

[33] Beck AT, Steer RA, Kovacs M, et al. Hopelessness and eventual suicide: a 10-year prospective study of patients hospitalized with suicidal ideation. Am J Psychiatry 1985 ;142(5):559-63.

[34] Bouvard M, Charles S, Guerin J, et al. [Study of Beck's hopelessness scale. Validation and factor analysis]. Encéphale. 1992 May-Jun;18(3):237-40.

[35] Kim CH, Jayathilake K, Meltzer HY. Hopelessness, neurocognitive function, and insight in schizophrenia: relationship to suicidal behavior. Schizophr Res. 2003 ;60(1):71-80.

[36] Westermeyer JF, Harrow M, Marengo JT. Risk for suicide in schizophrenia and other psychotic and nonpsychotic disorders. J Nerv Ment Dis. 1991 ;179(5):259-66.

[37] Beck AT, Brown G, Berchick RJ, et al. Relationship between hopelessness and ultimate suicide: a replication with psychiatric outpatients. Am J Psychiatry 1990 ;147(2):190-5.

[38] Glanz LM, Haas GL, Sweeney JA. Assessment of hopelessness in suicidal patients. Clinical Psychology Review 1995;15(1):49-64.

[39] Williams JB, Kobak KA. Development and reliability of a structured interview guide for the Montgomery Asberg Depression Rating Scale (SIGMA). Br J Psychiatry. 2008 ;192(1):52-8.

[40] Haatainen KM, Tanskanen A, Kylma J, et al. Life events are important in the course of hopelessness-a 2-year follow-up study in a general population. Soc Psychiatry Psychiatr Epidemiol. $2003 ; 38(8): 436-41$.

[41] Yip PS, Cheung YB. Quick assessment of hopelessness: a cross-sectional study. Health Qual Life Outcomes $2006 ; 4: 13$.

[42] McMillan D, Gilbody S, Beresford E, et al. Can we predict suicide and non-fatal self-harm with the Beck Hopelessness Scale? A meta-analysis. Psychol Med. 2007 ;37(6):769-78.

[43] Cochrane-Brink KA, Lofchy JS, Sakinofsky I. Clinical rating scales in suicide risk assessment. Gen Hosp Psychiatry. 2000 ;22(6):445-51.

[44] Ducher JL, Dalery J. [Concurrent validation of the suicidal risk assessment scale RSD with the Beck's suicidal ideation scale]. Encéphale. 2004;XXX:249-54. 
[45] Ducher JL, Terra JL. [Is the suicidal risk assessment scale RSD of predictive value?]. Encéphale. 2006 ;32(5 Pt 1):738-45.

[46] Ducher JL, Renoux M, Kwiatkowski F. Validation concourante de l'auto-questionnaire d'évaluation du risque suicidaire de Ducher aRSD. Poster 11ieme conférence l'Encéphale; Paris, France ; 2013. 